\title{
INTRODUCING ECOLOGICAL UNCERTAINTY IN RISK SENSITIVITY INDICES: THE CASE OF WIND FARM IMPACT ON BIRDS
}

\author{
Corrado Battistia ${ }^{a^{*}}$, Vincenzo Ferri ${ }^{\mathrm{b}}$, Luca Luiselli $^{\mathrm{c}, \mathrm{d}}$ and Giovanni Amori ${ }^{\mathrm{e}}$
}

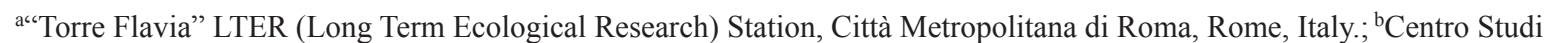
Naturalistici Arcadia, via Valverde 4, 01016 Tarquinia, Viterbo, Italy; drvincenzoferri@gmail.com; 'Institute for Development, Ecology, Conservation and Cooperation, via G. Tomasi di Lampedusa 33, I-00144 Rome, Italy, IT-00198 Rome, Italy; 1.luiselli@ ideccngo.org; ${ }^{\mathrm{d} D e p a r t m e n t ~ o f ~ A p p l i e d ~ a n d ~ E n v i r o n m e n t a l ~ B i o l o g y, ~ R i v e r s ~ S t a t e ~ U n i v e r s i t y ~ o f ~ S c i e n c e ~ a n d ~ T e c h n o l o g y, ~ P M B ~}$ 5080, Port Harcourt, Rivers State, Nigeria; ${ }^{\circ} \mathrm{CNR}$ Institute of Research on Terrestrial Ecosystems, viale dell'Università, 32 , I-00185 Rome, Italy; giovanni.amori@uniroma1.it

*Corresponding author. Email: c.battisti@cittametropolitanaroma.gov.it
\end{abstract}

\section{Article history}

Received: 21 October 2019; accepted 27 January 2020

\section{Keywords:}

Flight altitude; flight type; Shannon entropy; animal conservation

\begin{abstract}
In wind-farm impact assessments, it is useful to know the level of uncertainty that characterizes some key variables used to assess the sensitivity to risk in species of conservation concern. Here, we have introduced the use of the Shannon entropy as a value of ecological uncertainty of the prediction of the risk assessment index, obtained from two ecological traits (flight altitude and flight type). We based our evaluation of risk sensitivity on sampling of three common raptor birds (Gyps fulvus, Falco tinnunculus, Buteo buteo) all co-occurring in a wind farm landscape (central Italy). As to flight altitude, Gyps fulvus prefers the flight altitude category $>300 \mathrm{~m}$, Falco tinnunculus categorises closer to the ground and Buteo does not show a clear preference for any particular flight altitude category. As regards the flight type, Gyps fulvus showed significant preference for circular and thermal flight patterns, Falco tinnunculus for rapid horizontal/vertical flight patterns, while Buteo was found to prefer constant directions and circular and thermal flight patterns. Multiplication of the scores associated with these eco-behavioural traits by the number of recorded occurrences allowed estimation of risk sensitivity used to compute the risk assessment index. We normalized the partial scores of the risk associated with these traits with $\mathrm{H}^{\prime}$ values, thus obtaining more reliable speciesspecific normalized risk indices. The greater the entropy, the greater the level of uncertainty associated with it, and the lower the reliability of the risk index. Therefore, the entropy associated with flight behaviour diversity (altitude or type) could be a proxy for assessing uncertainty in wind power impact assessment. We think that normalizing indices of risk by including a measure of uncertainty can support decision makers in bird conservation and wind farm management.
\end{abstract}

\section{INTRODUCTION}

Disturbances are events that can disrupt any ecological level, or environmental component as well as the organizational status of a biological cycle of organisms (Pickett et al. 1989; Brawn et al. 2001; review in Battisti et al. 2016). Among anthropogenic disturbances (i.e. threats: Salafsky et al. 2008), wind farms are well known to impact birds in different ways (e.g., Winkelman 1992; Barrios and Rodrìguez 2004; Drewitt and Langston 2006; Johnson and Erickson 2010), birds of prey being particularly vulnerable to such disturbance factors (Madders and Whitfield 2006; Tellerìa 2009). Assessment of the wind farm impact on these vertebrates can be conducted either directly by recording carcasses lying under wind farm facilities (Drewitt and Langston 2008; Erickson et al. 2014) or indirectly (by assessing habitat displacements and changes in population parameters; Battisti et al. 2013). Moreover, it is also possible to highlight potential impacts by analyzing ecological and behavioural traits of the species that may or may not make individuals potentially exposed to these impacts (exposure to risk; Carrete et al. 2009). In this regard, there are several risk indices that are applied to wind farm impact assessment and to the identification of more sensitive species (Erickson et al. 2000; Garthe and Hüpop 2004; Noguera et al. 2010; Eichhorn et al. 2012). As regards certain ecological traits of the species that are considered to be sensitive to the risk of impact, the assessment of this risk is performed by assigning scores to categories of vulnerabilities selected a priori for specific variables. Some of these variables are linked to intrinsic factors (such as life-history traits derived from species ecology and behaviour, for example flight altitude or flight type, or anatomical features) or extrinsic (such as geo-morphological and vegetation characteristics of the territorial context; Noguera et al. 2010). In particular, risks of collision between raptors and wind farm blades are due to both the type and altitude of flight (PearceHiggins et al. 2009; Péron et al. 2017). Indeed, Noguera et al. (2010) estimated sensitivity to the potential risk by rating flight altitudes of various species (e.g. near 
the ground, under/near the blades $>15-30 \mathrm{~m}$; between the moving blades $>30-120 \mathrm{~m}$; above the blades $>$ $120 \mathrm{~m}$ ), considering that the height at which standard blades are located ranges approximately between 30 and $120 \mathrm{~m}$ above the ground. Therefore, the lowest scores were assigned to the flight altitude category that was more distant from rotor blade tips (e.g. 1 point to the flight altitude $>300 \mathrm{~m}$ above the ground) and progressively higher scores to altitude categories less distant from blade tips ( 2 points to flight altitudes $0-15 \mathrm{~m}$ and 150-300 m; 3 points to flight altitudes $15-30 \mathrm{~m}$ and $120-150 \mathrm{~m}$ above the ground), with the highest score (4) being assigned to the altitude category ranging between 30 and $120 \mathrm{~m}$, which corresponds to the operating range of rotor blades.

As regards the estimation of collision risks associated with the flight type, Noguera et al. (2010) gave the lowest scores (i.e. 1 point) to resting individuals, progressively increasing scores to individuals with constant direction flights ( 2 points), to birds whose flights are characterized by rapid wing movements ( 3 points) and, finally, to individuals with flights characterized by circular trajectories or following thermal wind pattern (maximum sensitivity/vulnerability 4 points). Once collision risks associated with both flight altitude and type had been assessed and rated in scores, the number of sightings recorded for flight altitude and type was multiplied by the relevant score. Thus, the sum of these values was considered to represent the partial risk sensitivity index that also includes evaluation of other eco-behavioural and conservation traits (e.g. seasonality, conservation status, density; Noguera et al. 2010). However, in these indices, which are based on summation or on averaged values, the uncertainty associated with the scores obtained (and so the predictive power of the index) is not taken into account. This may have implications for the assessment of the risk faced by each species. The uncertainty of a system is as high as its entropy is (Shannon and Weaver 1963).

Among the most common indices used for quantifying the entropy of a system is the Shannon-Wiener index (or Shannon entropy index, see Jost 2006). This measure was originally used to quantify the entropy, a proxy of uncertainty (or level of surprise), in an information context. Therefore, the Shannon entropy quantifies the uncertainty associated with prediction (see also Magurran 2004; Ricotta 2005; Magurran and McGill 2011): the higher the value of the Shannon entropy index, the more difficult is correct prediction of the process underlying the data.

In this paper, we have introduced the use of the Shannon entropy as a value of prediction uncertainty obtained from two components (flight altitude and flight type) of the risk assessment index. We based our evaluation of uncertainty on the original sampling data for three raptor species (Aves, Falconiformes) co-occurring in a large wind farm landscape in central Italy. We think that inclusion of an uncertainty measure in the assessment carried out with risk sensitivity indices can support decision makers in bird conservation and wind farm management.

\section{MATERIALS AND METHODS}

\section{Study area}

This study was conducted in an approximately $35 \mathrm{~km}^{2}-$ large area, which is located near the Fucino Valley and the Sirente-Velino Natural Regional Park, along the southern slopes of the Sirente Massif, with an altitudinal range of 900-1200 m a.s.l. It includes the municipalities of Cerchio, Collarmele and Pescina (Province of L'Aquila; central Italy; WGS 84, F33, UTM coordinates: top left - X 381.985, Y 4.663.406; bottom right - X 397.520, Y 4.653.612). The wind farm in this study contained 44 one-bladed turbines (rated power output of 250 and $350 \mathrm{~kW}$; CerchioCollarmele-Pescina wind farm or CCP) erected between 1992 and 2005. In 2007, older turbines were removed and repowered with 17 three-bladed turbines (Vestas V80 rated for 2.0 MW; ENECO Power Station). In the period 2007-2009, there were also two meteorological towers at the wind farm, one of which was $30 \mathrm{~m}$ and another $50 \mathrm{~m}$ high. The rotor diameter of each three-blade turbine was $80 \mathrm{~m}$, the rotor span area was $5072 \mathrm{~m}^{2}$ and the tubular steel tower was $78 \mathrm{~m}$ high. Tips of the turbine blades reached the maximum height of $117 \mathrm{~m}$ above the ground level. The Vestas turbines on the CCP wind farm were located in a heterogeneous mosaic-like landscape characterized by hemi-cryptophytic pastures (Brachypodium rupestre dominant) with nitrophilous phyto-coenoses and sparse brushes (for cattle presence) at altitudes ranging between 970 and $1160 \mathrm{~m}$ a.s.l. (Pirone and Tammaro 1997). We considered this study area to be a unique sampling area because of the uniformity of landscape characteristics. The local climate is cold temperate. In the course of the study, no remarkable changes in mean temperatures and rain regimes were observed (ENECO Meteorological Station, Collarmele; www.meteo.it: Valle del FucinoAvezzano).

\section{Protocol}

The overall survey included collection, analysis and interpretation of the data relating to the presence of a group of raptor species particularly sensitive to the presence of wind power plants (Falconiformes) in the study area.

Duration of the field sampling that was conducted in the study area from 2011 to 2014 was approximately 
304 hours (2011: 2 April - 20 May, 21 September 31 December; 2012: 1 January - 3 August; 2013: 14 May - 16 June; 2014: 9 March - 9 September). During each visit, we collected data on the presence of vagrant species, with both flight altitude and flight type of each individual recorded:

(i) Flight altitude (taking into account the following altitudinal classes (above the ground) in metres: $0-15,>15-30,>30-120,>120-150$, $>150-300,>300$, from Noguera et al. 2010, modified). These altitudinal classes were singled out because they corresponded to specific areas of turbine operation, thus representing the height bands differing in potential risk to birds (under the blades: $0-15 \mathrm{~m}$ and $>15-30 \mathrm{~m}$, between the blades in movement: $>30-120 \mathrm{~m}$ and above the blades: $>120 \mathrm{~m}$, the swept area of turbine blades is within the height range $30-120 \mathrm{~m}$ ). The flight altitude was determined taking into account the reference points present on the wind facilities. The flight altitude was rated in terms of potential risk as follows: Score 1 was assigned to the sightings of raptors at heights $>300 \mathrm{~m}$ above the ground; 2 points were given to the sightings of birds flying at heights $0-15 \mathrm{~m}$ and $150-300 \mathrm{~m} ; 3$ points were given to bird sightings at heights $15-30 \mathrm{~m}$ and $120-150 \mathrm{~m}$; and 4 to the sightings of raptors at heights $30-120 \mathrm{~m}$ above the ground.

(ii) Type of flight. In accordance with Noguera et al. (2010), different types of flight were rated in terms of potential risk as follows: laid flights -1 point; flights in a constant direction -2 points; flights with rapid vertical/horizontal flight wing movements -3 points; flights following circular trajectories and/or thermal winds -4 points (maximum sensitivity).

Both for the altitude and type of flight, the number of records was multiplied by the relevant scores and the total value obtained was considered to represent the partial risk score (partial risk score associated with flight altitude is further referred to as FAps, and the partial risk score associated with the type of flight is referred to as TFps) that could be included in a general risk sensitivity index.

For observations, we used a two-colour Pentax model No.574 10x50 FIELD 5.5 ${ }^{\circ}$, with Coated - Mod. Optics zoom 8-20 $\times 50$ and an telescope (Kowa Model TSN-1 multicoated lens zoom 20-60×).

\section{Data analyses}

The relative frequency $\left(\mathrm{fr}_{\mathrm{CAT}}\right)$ of each flight altitude category and that of each flight type category were calculated.
From the flight altitude and flight type frequency data, Shannon entropy (Magurran 2004) was calculated as follows:

$$
\mathrm{H}^{\prime}=-\Sigma \mathrm{fr}_{\mathrm{CAT}} \ln \mathrm{fr}_{\mathrm{CAT}}
$$

The calculated value of the entropy index for flight altitude $\left(\mathrm{H}_{\mathrm{FA}}^{\prime}\right)$ and for type of flight $\left(\mathrm{H}_{\mathrm{TF}}^{\prime}\right)$ ranges between 0 and $\mathrm{n}$. The higher the value, the higher the entropy level of the associated information (i.e. the greater the associated uncertainty in the evaluation) is.

To obtain the uncertainty - normalized risk index (URI) for each trait, we calculated the ratio FAps/ $\mathrm{H}_{\mathrm{FA}}^{\prime}$, for flight altitude, and TFps/ $\mathrm{H}_{\mathrm{TF}}$ for the type of flight, thus obtaining two partial indices (respectively $\mathrm{URI}_{\mathrm{FA}}$ and $\mathrm{URI}_{\mathrm{TF}}$ ). Statistical differences between URI indices for the three species studied were assessed by one-way ANOVA.

Comparisons between frequency values (between categories and species) were made by $\chi^{2}$ test. Alpha was set at 5\%. We used SPSS 13.0 for Windows and Primer 4.2 software for statistical analyses.

\section{RESULTS}

Overall, 907 records were obtained regarding flight altitudes, and 667 regarding flight types, for a total of 17 Falconiformes species. As regards the most common sedentary species, for which a representative contact sample (n > 20) was obtained (Gyps fulvus, Falco tinnunculus and Buteo buteo), 742 records were obtained for flight altitudes and 648 for flight types.

As to the flight altitude (Table 1), Gyps fulvus records were mostly related to the altitude category $>300 \mathrm{~m}$ (with significant differences; $\chi^{2}=69.192 ; p=0.001$ ), while those of Falco tinnunculus to the altitude categories closer to the ground (from 0-15 $\mathrm{m}$ to $30-120 \mathrm{~m}$; $\chi^{2}=75.056 ; p=0.001$ ), and Buteo buteo records did not differ significantly in terms of frequencies of flight altitude categories $\left(\chi^{2}=2.426 ; p=0.670\right)$.

As regards the flight type (Table 2), Gyps fulvus records showed the highest frequency in the category "circular and thermal flight patterns"; $\chi^{2}=165.064, p=0.001$ ), Falco tinnunculus records in the category "rapid flight patterns" $\left(\chi^{2}=34.575, p=0.001\right)$, while those of Buteo buteo in the flight type categories "constant direction" and "circular and thermal flight patterns" $\left(\chi^{2}=11.201\right.$, $p=0.001$ ).

As to the flight altitude, the lowest values of the Shannon entropy H' were recorded in Falco tinnunculus (Table 1), but this species showed the highest value in this metric when the flight type was considered (Table 2). Falco tinnunculus showed the highest value in 
Table 1. Flight altitude categories, score of the risk assigned, number (N) and relative frequency (Fr) of total records and total scores for each category and each species (period 2011/2014). Averaged total scores (FAps), Shannon-Wiener index ( $\left.{ }^{\prime}{ }_{\mathrm{FA}}\right)$ and uncertainty normalized risk index $\left(\mathrm{URI}_{\mathrm{FA}}\right)$ values for each species.

\begin{tabular}{|l|c|c|c|c|c|c|c|c|c|c|}
\hline & & \multicolumn{3}{|c|}{ Gyps fulvus } & \multicolumn{3}{c|}{ Falco tinnunculus } & \multicolumn{3}{c|}{ Buteo buteo } \\
\hline Flight altitude category & Score & $\mathrm{N}$ & $\mathrm{Fr}$ & Score & $\mathrm{N}$ & Fr & Score & $\mathrm{N}$ & Fr & Score \\
\hline $0-15$ & 2 & 74 & 0.134 & 148 & 46 & 0.286 & 92 & 4 & 0.143 & 8 \\
\hline $15-30$ & 3 & 69 & 0.125 & 207 & 49 & 0.304 & 147 & 6 & 0.214 & 18 \\
\hline $30-120$ & 4 & 70 & 0.127 & 280 & 42 & 0.261 & 168 & 7 & 0.250 & 28 \\
\hline $120-150$ & 3 & 71 & 0.128 & 213 & 17 & 0.106 & 51 & 3 & 0.107 & 9 \\
\hline $150-300$ & 2 & 98 & 0.177 & 196 & 6 & 0.037 & 12 & 5 & 0.179 & 10 \\
\hline 300 & 1 & 171 & 0.309 & 171 & 1 & 0.006 & 1 & 3 & 0.107 & 3 \\
\hline Total & & 553 & 1.000 & 1215 & 161 & 1.000 & 471 & 28 & 1.000 & 76 \\
\hline FAps & & & & 2.197 & & & 2.925 & & & 2.714 \\
\hline H $_{\text {FA }}$ & & & 1.724 & & & 1.461 & & & 1.741 & \\
\hline URI $_{\text {FA }}$ & & & 1.274 & & & 2.002 & & & 1.559 & \\
\hline
\end{tabular}

Table 2. Type of flight categories, score of the risk assigned, number (N) and relative frequency (Fr) of total records and total scores for each category and each species (period 2011/2014). Averaged total scores (TFps), Shannon-Wiener index ( $\mathrm{H}_{\mathrm{TF}}$ ) and uncertainty normalized risk index $\left(\mathrm{URI}_{\mathrm{TF}}\right)$ values for each species.

\begin{tabular}{|l|c|c|c|c|c|c|c|c|c|c|}
\hline & & \multicolumn{3}{c|}{ Gyps fulvus } & \multicolumn{3}{c|}{ Falco tinnunculus } & \multicolumn{3}{c|}{ Buteo buteo } \\
\hline \multicolumn{1}{|c|}{ Flight type } & Score & $\mathrm{N}$ & $\mathrm{Fr}$ & Score & $\mathrm{N}$ & Fr & Score & $\mathrm{N}$ & Fr & Score \\
\hline Laid & 1 & 55 & 0.14 & 55 & 10 & 0.07 & 10 & 1 & 0.04 & 1 \\
\hline Constant direction & 2 & 127 & 0.33 & 254 & 35 & 0.26 & 70 & 10 & 0.42 & 20 \\
\hline $\begin{array}{l}\text { Rapid horizontal/ } \\
\text { vertical movements }\end{array}$ & 3 & 10 & 0.03 & 30 & 64 & 0.47 & 192 & 2 & 0.08 & 6 \\
\hline Circular/thermal flights & 4 & 195 & 0.50 & 780 & 28 & 0.20 & 112 & 11 & 0.46 & 44 \\
\hline Total & & 387 & 1.00 & 1119 & 137 & 1.00 & 384 & 24 & 1.00 & 71 \\
\hline TFps & & & & 2.891 & & & 2.803 & & & 2.958 \\
\hline $\mathrm{H}_{\text {TF }}$ & & & 1.093 & & & 1.753 & & & 1.052 & \\
\hline URI $_{\text {TF }}$ & & & 2.645 & & & 1.599 & & & 2.812 & \\
\hline
\end{tabular}

partial $\mathrm{URI}_{\mathrm{FA}}$ (Table 1) and the lowest value in $\mathrm{URI}_{\mathrm{TF}}$ (Table 2). Interspecific differences in both $\mathrm{URI}_{\mathrm{FA}}$ and $\mathrm{URI}_{\mathrm{TF}}$ were statistically significant (one-way ANOVA, $\mathrm{URI}_{\mathrm{FA}}: \mathrm{F}_{2,18}=11.98, p<0.001 ; \mathrm{URI}_{\mathrm{TF}}: \mathrm{F}_{2,12}=15.17$, $p<0.001$ ) (Tukey's HSD post-hoc revealing that Gyps fulvus was different from the other two species).

\section{DISCUSSION}

In our case study, the three species of Falconiformes showed different behaviour in terms of flight altitude and flight type. Namely, Gyps fulvus was frequently observed flying in a constant direction at high flight altitudes; Falco tinnunculus was frequently sighted at low-medium altitudes (up to $120 \mathrm{~m}$ above the ground), its flight behaviour being characterized mainly by rapid horizontal/ vertical flight patterns; and Buteo buteo did not show a preference for any particular flight altitude, its flight being characterized by a constant direction and circular/ thermal flight patterns. Once the risk scores assigned to these flying traits had been multiplied by the number of recorded occurrences, a higher partial risk score was obtained for Falco tinnunculus and Buteo buteo and a lower one for Gyps fulvus. However, if Buteo buteo and Gyps fulvus showed high Shannon entropy, the values of this index for Falco tinnunculus were low, thus supporting the empirical risk score for this species, the same being not true for the two previous species.

As regards the flight type, the observed risk scores for all the three species (ranging between 2.80-2.96) were similar. Still, the Shannon entropy appeared to be very different with Falco tinnunculus showing the highest values, thus evidencing that the risk score for this species is weak in predictive power. Once the averaged partial scores had been normalized with the Shannon entropy, partial URI for both traits in each species were obtained. This normalization using the amount of uncertainty associated with different flight altitude and type of flight has changed the ranking of the risk level faced by each species. For example, as regards the type of flight, after normalization, the apparent similarity in partial score values among the investigated species (ranging between 2.80 and 2.96) abruptly ended: Buteo buteo showed the highest partial URI (2.81), while Falco tinnunculus the lowest (1.60).

Estimates of the entropy associated with flight altitude and/ or flight type could be considered as proxies for assessing 
uncertainty in wind power impact assessment. In windfarm impact assessments, it is useful to know the level of uncertainty that characterizes the outputs of some key variables used as proxies to assess the risk sensitivity of species of ecological value and conservation concern.

The frequency associated with a particular altitude or type of flight, appropriately weighed with a risk score, may be associated with the level of impact risk. Nevertheless, we suggest that the levels of uncertainty expressed in connection with the variable taken into consideration should be associated with risk index values. This uncertainty can be a proxy for the non-reliability of the index itself.

We think that this approach can also be applied to other targets that could be impacted by wind farms, e.g. other large-sized bird species, for example, Charadriiformes and Gruiformes (e.g. Stewart et al. 2007; Zieliński et al. 2007) and bats (Chiroptera; Trille et al. 2008; Jain et al. 2010; Ferri et al. 2016), for which, it could be useful to obtain risk indices based on ecological traits. In this regard, further field and analytical research is necessary.

\section{COMPETING INTEREST}

The authors declare that they have no competing interest.

\section{ACKNOWLEDGMENTS}

We wish to thank Nicoletta Conte, Osvaldo Locasciulli and the staff of the ENECO Wind Farm Power Station of Collarmele (L'Aquila) for support and suggestions. A. Zocchi reviewed the English style and language. An anonymous reviewer, Laima Monkiene and the Editor largely improved the first draft of the manuscript providing useful comments and suggestions.

\section{ORCID: 0000-0002-2621-3659}

\section{DISCLOSURE STATEMENT}

No potential conflict of interest was reported by the authors.

\section{REFERENCES}

Barrios, L., and A. Rodrìguez. 2001. Behavioural and environmental correlates of soaring-bird mortality at on-shore wind turbines. Journal of Applied Ecology 41: 72-81.

Battisti, C., D. Franco, C. Norscia, P. Santone, C. Soccini, and V. Ferri. 2013. Estimating the indirect impact of wind farms on breeding bird assemblages: a case study in the central Apennines. Israel Journal of Ecology and Evolution 59: 125-129.

Battisti, C., G. Poeta, and G. Fanelli. 2016. An introduction to Disturbance Ecology. A road map for wildlife management and conservation. Switzerland: Springer.

Brawn, J. D., S. K. Robinson, and F. R. Thompson III. 2001. The role of disturbance in the ecology and conservation of birds. Annual Review of Ecology and Systematic 32: 251-276.

Carrete, M., J. A. Sánchez-Zapata, J. R. Benítez, M. Lobón, and J. A. Donázar. 2009. Large scale risk-assessment of wind-farms on population viability of a globally endangered long-lived raptor. Biological Conservation 142: 2954-2961.

Drewitt, A. L., and R. H. Langston. 2006. Assessing the impacts of wind farms on birds. Ibis 148 (s1): 29-42.

Drewitt, A. L., and R. H. Langston. 2008. Collision effects of wind-power generators and other obstacles on birds. Annals New York Academy of Sciences 1134: 233-266.

Eichhorn, M., K. Johst, R. Seppelt, and M. Drechsler. 2012. Model-based estimation of collision risks of predatory birds with wind turbines. Ecology and Society 17 (2): 1.

Erickson, W. P., M. D. Strickland, G. D. Johnson, and J. W. Kern. 2000. Examples of statistical methods to assess risk of impacts to birds from wind plants. In Proceedings of the Avian-Wind Power Planning Meeting III, RESOLVE, Inc., Technical coordinator, 172-182. Washington, DC, USA: National Wind Coordinating Committee.

Erickson, W. P., M. M. Wolfe, K. J. Bay, D. H. Johnson, and J. L. Gehring. 2014. A comprehensive analysis of small-passerine fatalities from collision with turbines at wind energy facilities. PloS One 9: e107491.

Ferri, V., C. Battisti, and C. Soccini. 2016. Bats in a Mediterranean Mountainous Landscape: Does Wind Farm Repowering Induce Changes at Assemblage and Species Level? Environmental Management 57: 1240-1246.

Garthe, E., and O. Hüppop. 2004. Scaling possible adverse effects of marine wind farms on seabirds: developing and applying a vulnerability index. Journal of Applied Ecology 41: 724-734.

Jain, A. A., R. R. Koford, A. W. Hancock, and G. G. Zenner. 2010. Bat mortality and activity at a Northern Iowa wind resource area. American Midland Naturalist 165: 185-200.

Johnson, G. D., and W. P. Erickson. 2010. Avian, bat and habitat cumulative impacts associated with wind energy development in the Columbia Plateau Ecoregion of Eastern Washington and Oregon. Klickitat County 
Planning Department, Western EcoSystems Technology, Inc.

Jost, L. 2006. Entropy and diversity. Oikos 113: 363-375.

Magurran, A. 2004. Measuring biological diversity. Malden: Blackwell Publishing.

Madders, M., and D. P. Whitfield. 2006. Upland raptors and the assessment of wind farm impacts. Ibis 148 (s1): 43-56.

Magurran, A., and B. J. McGill. 2011. Biological diversity. Frontiers in measurements and assessments. Oxford, New York: Oxford University Press.

Noguera, J. C., I. Pérez, and E. Minguez. 2010. Impact of terrestrial wind farms on diurnal raptors developing a spatial vulnerability index and potential vulnerability maps. Ardeola 57: 41-53.

Pearce-Higgins, J. W., L. Stephen, R. H. Langston, I. P. Bainbridge, and R. Bullman. 2009. The distribution of breeding birds around upland wind farms. Journal of Applied Ecology 46: 1323-1331.

Péron, G., Fleming, C. H., O. Duriez, J. Fluhr, C. Itty, S. Lambertucci, K. Safi, E. L. C. Shepard, and J. M. Calabrese. 2017. The energy landscape predicts flight altitude and wind turbine collision hazard in three species of large soaring raptor. Journal of Applied Ecology, in press.

Pickett, S. T. A., J. Kolasa, J. J. Armesto, and S. L. Collins. 1989. The ecological concept of disturbance and its expression at various hierarchical levels. Oikos 54: 129-136.

Pirone, G., and F. Tammaro. 1997. The hilly calciophilous garigues in Abruzzo (Central Apennines - Italy). Fitosociologia 32: 73-90.
Ricotta, C. 2005. Through the jungle of biological diversity. Acta Biotheoretica 53: 29-38.

Salafsky, N., N. Salzer, A. J. Stattersfield, C. Hilton-Taylor, R. Neugarten, S. H. M. Butchart, B. Collen, N. Cox, L. L. Master, S. O'Connor, and D. Wilkie. 2008. A standard lexicon for biodiversity conservation: unified classifications of threats and actions. Conservation Biology 22: 897-911.

Shannon, C. E., and W. Weaver. 1963. Mathematical theory of communication. Urbana, Illinois: University of Illinois Press.

Stewart, G. B., A. S. Pullin, and C. F. Coles. 2007. Poor evidence-base for assessment of windfarm impacts on birds. Environmental Conservation 34 (1): 1-11.

Tellerìa, J. L. 2009. Overlap between wind power plants and Griffon Vultures Gyps fulvus in Spain. Bird Study 56: $268-271$.

Trille, M., R. Liozon, and S. Talhoët. 2008. Suivi ornithologique et chiroptérologique du parc éolién de Castelnau-Pégayrols. Bilan de la première année de suivi. Lpo-aveyron.

Winkelman, J. E. 1992. The impact of the Sep wind park near Oosterbierum (Fr.), the Netherlands, on birds, 1: collision victims. DLO Instituut voor Bos-en Natuuronderzoek. RINrapport 92/3. 4 volumes.

Zieliński, P., G. Bela, and K. Kwitowski. 2007. Report on monitoring influence of wind farm operating near Gniezdżewo (gmina of Puck, pomorskie voivodeship) on birds. Dipol sp. z. o.o. Available on: https://www. netinform.net/KE/files/pdf/Puck_Annual\%20Monitoring\%20Report_2007_Annex\%20no\%202.pdf 